\title{
Warrant Pricing Using Observable Variables
}

\author{
Andrey D. Ukhov* \\ Kelley School of Business, Indiana University
}

14 August 2003

\begin{abstract}
The classical warrant pricing formula requires knowledge of the variance of the firm value process, and the firm value. When warrants are outstanding the firm value itself is a function of the warrant price. Firm value and the variance of the firm value are then unobservable variables. I develop an algorithm for pricing warrants using stock prices, an observable variable, and variance of stock returns. The method also enables estimation of the variance of firm value. A proof of existence of the solution is provided.
\end{abstract}

JEL Classification: G13, G63.

Keywords: warrant pricing, contingent claims pricing.

\footnotetext{
${ }^{*}$ I would like to thank Jonathan E. Ingersoll, Jr., William T. Moore (the editor), Matthew Spiegel, and an anonymous referee for helpful comments. Mailing address: Andrey D. Ukhov, Kelley School of Business, Indiana University, 1309 East 10th Street, Bloomington, IN 47405. Telephone: 812-855-2698, Fax: 812-855-5875. E-mail: aukhov@indiana.edu
} 


\section{Introduction}

Rights and warrants issued by a firm on its own equity are different from call options because the exercise price is paid to the firm and increases its assets, and new shares of stock are issued at exercise. Warrant pricing is more complicated than option pricing, and requires adjustments to values computed according to an option pricing model. When a warrant is exercised, new shares of stock are issued, and the cash payment that is made increases the assets of the issuing firm. ${ }^{1}$ Correction for dilution was first proposed by Galai and Schneller (1978), but two issues remain unresolved. The classical Black-Scholes style warrant pricing formula (Galai and Schneller (1978), Ingersoll (1987), and Hull (2003)) requires knowledge of the firm value. The firm value itself, however, is a function of the warrant price and therefore is unobservable.

The formula also requires the variance of the value process. The variance cannot be estimated directly since the value process itself is not observed. Stock prices and stock volatility, however, may be observed. For example, when a firm prices a new warrant issue, it can use the current price of its stock and the stock volatility estimated from historical stock prices. The same problem arises when valuing warrants that are privately held. In this case, only the volatility and the value of the stock of the firm are observed, not the value and the volatility of the firm equity value. Several authors remark on these difficulties (Galai and Schneller (1978), Crouhy and Galai (1991), and Veld (2000)), but so far no solution has been proposed.

I develop an algorithm for pricing warrants using only observable variables, and it is the first such algorithm published to my knowledge. The method does not rely on an approximation of the

\footnotetext{
${ }^{1}$ Phelps, Moore, and Roenfeldt (1991) show that warrants can represent potentially large additions to outstanding equity. In their sample, the median ratio of the number of new shares issued in the event of full exercise to the number of shares outstanding before issuance is 18 percent.
} 
value process or a guess about the volatility of the firm value. Instead, a system of two non-linear equations is shown to describe the relationship between the observed variables, the stock price and the estimated variance of stock returns, and warrant value and the volatility of the firm. It is shown that the system always admits a solution. This system of non-linear equations is no more difficult to program in any conventional software than the original Black-Scholes option pricing formula. The equations are well-behaved and do not require special numerical methods.

A benefit arising from the method is that the algorithm brings into focus the effect that a warrant issuance has on the volatility of the firm value, because it simultaneously computes warrant price and the estimate of the firm value volatility. It is assumed throughout the paper that the volatility of the firm is constant. The volatility of the firm is a weighted average of the volatilities of stocks and warrants. When stock price changes, the relative contribution of the stock volatility to the firm value volatility changes, as well. Since the warrant is riskier than the stock, the volatility of the stock is less than the volatility of the firm. The difference in volatility in warrant pricing and option pricing is noted by Veld and Verboven (1995) who study implied volatilities of warrants and long term call options written on the same stock, N.V. Philips. They find higher implied volatilities for warrants than for long term call options.

\section{Warrant Pricing Algorithm}

Consider valuation of a conventional warrant, issued by a firm for its own stock. ${ }^{2}$ Suppose that a company has $N$ shares of common stock and $n$ warrants outstanding. Each warrant entitles the owner to receive $k$ shares of stock upon payment of $X$ dollars. These two forms of financing are

\footnotetext{
${ }^{2}$ Rights offerings which create new shares of stock when exercised can also be priced by this algorithm. Like warrants, rights are also issued by a firm.
} 
the only ones that the company is using. Let $V$ be the value of the company's assets.

Let $\mathcal{W}(V, \tau)$ denote the value of each warrant on a firm of value $V$ when the time to maturity is $\tau$. The warrants are exercised only if $k S \geq X$, just as with call options. When all $n$ warrants are exercised the firm receives $n X$ and issues $k n$ new shares of stock. If the Black-Scholes assumptions are satisfied, the value of the warrant is ${ }^{3}$

$$
\begin{aligned}
\mathcal{W}(V, \tau ; X, \sigma, r, k, N, n) & =\frac{1}{N+k n} \mathcal{C}(k V, \tau ; N X, \sigma, r) \\
& =\frac{1}{N+k n}\left[k V \cdot \Phi\left(\eta_{W}\right)-e^{-r \tau} \cdot N X \cdot \Phi\left(\eta_{W}-\sigma \sqrt{\tau}\right)\right] \\
\text { where } \eta_{W} & \equiv \frac{\ln (k V / N X)+\left(r+\frac{1}{2} \sigma^{2}\right) \tau}{\sigma \sqrt{\tau}} .
\end{aligned}
$$

In equation $(1), \mathcal{C}(\cdot)$ is the Black-Scholes call option valuation formula, $\tau$ is the remaining time until maturity in years, $r$ is the continuously compounded interest rate, $e^{r}=R, R$ is one plus the annual interest rate, $\sigma$ is the annual standard deviation in (logarithmic) returns on the value process, and $\Phi(\cdot)$ is the cumulative normal distribution function. ${ }^{4}$

Two comments on this well-known warrant valuation formula are in order. Suppose the task is to price a warrant that will be issued soon. Current stock price, $S$, and the number of shares outstanding, $N$, are observed. However, the formula (1) requires as an input the value of the firm, $V$, which will include the value of all warrants and is thus unknown. It is assumed in (1) that the value of the firm, $V$, follows a log-normal distribution and the parameter $\sigma$ is the annual standard deviation of the (logarithmic) return on the value process, $V$. Since $V$ is not observed, $\sigma$ cannot be estimated directly. What can be estimated from the historical stock prices is the volatility of

\footnotetext{
${ }^{3}$ Galai and Schneller (1978), Ingersoll (1987) and Ingersoll (1994).

${ }^{4}$ The formula in (1) can be used to price the warrants even if there are more than only warrants and common stock in the capital structure of the firm. In this case $V$ is the market value of just these two components. If $V$ has a lognormal distribution than the Black-Scholes formula is valid (Ingersoll (1987)).
} 
the stock, $\sigma_{S}$, before the warrant issuance.

We assume that the volatility of the firm, $\sigma$, is constant. Warrants and stocks are contingent claims on the value of the firm. The volatility of the firm is a weighted average of the volatilities of the two claims. Since the warrant is riskier than the stock, the volatility of the stock is less than the volatility of the entire firm. Define $\Omega_{S} \equiv \frac{\triangle_{S} V}{S}$, the elasticity of the stock price with respect to the firm value, where $\triangle_{S}$ is a hedge ratio for the stock. Hedge ratio $\triangle_{S}$ measures the dollar price change for the share of stock when the value of the entire firm changes by one dollar. The volatility of the stock is related to the volatility of the firm via ${ }^{5}$

$$
\sigma_{S}=\Omega_{S} \cdot \sigma=\left(V \triangle_{S} / S\right) \cdot \sigma \Leftrightarrow \sigma=\frac{\sigma_{S}}{V \triangle_{S} / S}
$$

To compute $\triangle_{S}$ first compute $\triangle_{W}$, the hedge ratio for a warrant. For a warrant, $\triangle_{W}$ is the delta measuring the dollar change in warrant price for a one dollar change in the value of the firm. If equation (1) is used, then delta $\left(\triangle_{W}\right)$ that measures the dollar change in warrant price for a one dollar change in the value of the firm is

$$
\triangle_{W}=\frac{\partial \mathcal{W}(V ; \bullet)}{\partial V}=\frac{k}{N+k n} \Phi\left(\eta_{W}\right)
$$

In our model, a share of stock is also a contingent claim on the whole firm. Hence, it too has a hedge ratio that measures the dollar stock price change when the value of the firm, $V$, changes by one dollar. When the value of the firm changes by $\$ 1$, the warrant's price changes by $\triangle_{W}$ and the stock price changes by $\triangle_{S}$. Then $n \cdot \triangle_{W}+N \cdot \triangle_{S}=\triangle_{V}=1$, and therefore

$$
\triangle_{S}=\frac{1-n \cdot \triangle_{W}}{N}=\frac{N+k n-n k \Phi\left(\eta_{W}\right)}{N(N+k n)}
$$

We are now ready to state the method for computing warrant price from the observed variables.

\footnotetext{
${ }^{5}$ For example, Ingersoll (1994).
} 
Algorithm 1 To compute warrant price using the observed stock price $S$, and the estimate of stock volatility $\sigma_{S}$,

1. Solve (numerically) the following system of non-linear equations for $\left(V^{*}, \sigma^{*}\right)$,

$$
\left\{\begin{array}{l}
S \cdot N=V-n \cdot \mathcal{W}(V, \tau ; X, \sigma, r, k, N, n) \\
\sigma_{S}=\left(V \triangle_{S} / S\right) \cdot \sigma
\end{array}\right.
$$

where $\triangle_{S}$ is a function of $\eta_{W}$, and therefore a function of $V$ and $\sigma$.

2. The warrant price, $w$, is obtained as

$$
w=\frac{V^{*}-S \cdot N}{n}
$$

It is important to prove that the system (5) admits a solution.

Theorem 1 The system (5) in Algorithm 1 has a solution $\left(\sigma^{*}, V^{*}\right) \in(0,+\infty) \times(0,+\infty)$.

The proof of Theorem 1 consists of several steps that are presented in the Appendix. In the first step, it is shown that the first equation of the nonlinear system (5) defines a strictly positive monotonically increasing continuous function $\psi_{1}(\sigma)=V$. This function relates the value of the firm, $V$, to the variance $\sigma$. The second step proves that the second equation of the nonlinear system (5) defines another relationship between $\sigma$ and $V, \psi_{2}: \sigma \mapsto V$. In the third step we examine the range of values attained by $\psi_{1}$ and $\psi_{2}$ to prove that they intersect.

\section{Examples}

I will now show how to use the algorithm to price warrants issued by Expedia, Inc., an on-line travel services company. Expedia is listed on NASDAQ (symbol EXPE) since November 10, 1999. 
On February 5, 2002 Expedia issued warrants (traded under symbol EXPEW). I will price the warrant on the issue date. At issue, the company had 25.412 million shares outstanding, issued 3.2 million warrants, each warrant entitled the owner to receive 1 share of Expedia upon payment of $52 \operatorname{dollars}(N=25.412$ million, $n=3,200,000, k=1, X=52){ }^{6}$ This was a seven-year warrant $(\tau=7)$ expiring on February 4, 2009. The company paid no dividends. At issue date, the annual rate on a 7-year Treasury was $4.4 \%(r=\ln 1.044)$. Expedia's stock price on February 5, 2002 was $S=24.65$. I use daily stock prices from 11 November 1999 until February 4, 2002 to estimate annualized stock volatility $\sigma_{S}=155 \%$ (historical volatility on Expedia has been very high).

First, we value the warrant approximately, as a regular call option, using Black-Scholes formula. The value computed under this approximation is

$$
\begin{aligned}
w_{\text {Call }} & =\mathcal{C}\left(S, \tau ; X, \sigma_{S}, r\right) \\
& =S \cdot \Phi\left(\eta_{\text {Call }}\right)-e^{-r \tau} \cdot X \cdot \Phi\left(\eta_{W}-\sigma \sqrt{\tau}\right), \\
\text { where } \eta_{\text {Call }} & \equiv \frac{\ln (S / X)+\left(r+\frac{1}{2} \sigma_{S}^{2}\right) \tau}{\sigma_{S} \sqrt{\tau}} .
\end{aligned}
$$

Using the above parameter values, $w_{\text {Call }}=23.41$.

Second, we can use (1) and approximate $\sigma \approx \sigma_{S}$, and $V \approx S \cdot N$ to compute the warrant value $w_{W}$. For the above parameter values, $w_{W}=20.80$.

Finally, we compute the value of the warrant using Algorithm 1. Then, according to (5), we

\footnotetext{
${ }^{6}$ The terms of the warrants changed on March 11, 2003 due to the 2 for 1 stock split of EXPE. The new strike price was set at 26 dollars $(X=26)$, each warrant was split into two $(n=6,400,000)$, and there were 50.824 million shares outstanding after the split. We are pricing the warrant before this split, however, and thus we are using pre-split numbers. The after-split figures are given here for a more complete description of the contract.
} 
solve the following system,

$$
\left\{\begin{array}{l}
24.65 \cdot 25.412=V-3.2 \cdot \mathcal{W}(V, \tau ; X, \sigma, r, k, N, n) \\
155 \%=\left(V \triangle_{S} / 24.65\right) \cdot \sigma
\end{array}\right.
$$

to obtain values of $V$ and $\sigma$. In this system, $\tau, X, r, k, N$, and $n$ are problem parameters. The delta of the stock, $\triangle_{S}$, also depends on $V, \sigma$, and the problem parameters. Solving the system numerically, we obtain the value of Expedia's warrant $w_{A}=23.36$ and the variance of the firm value, $\sigma=155.44 \%$.

Warrant prices obtained by three valuation methods are close to each other in case of Expedia's warrant. Next, we investigate whether they are close for hypothetical warrants with different contract characteristics. Table 1 shows warrant prices computed by three methods for different warrants. Parameters common for all calculations are: $k=1, X=100, \tau=3, r=0.0488$, $N=100$. Other parameters, including the stock price, the number of warrants outstanding, and the variance of stock returns vary in the table.

As dilution increases, the magnitude of the difference between warrant prices computed by different methods increases. This can be seen by comparing columns $w_{\text {Call }}$ and $w_{A}$ in Table 1 for the low dilution and the high dilution cases. For $\sigma_{S}=25 \%$, in the high dilution case the difference in value between $w_{C a l l}$ and $w_{A}$ equals $4.3 \%$ for the out-of-the-money warrant $(S=75)$. For a higher stock variance, $\sigma_{S}=40 \%$, the difference in value between $w_{C a l l}$ and $w_{A}$ in the high dilution case decreases to $2.8 \%$.

The difference in prices is higher for out-of-the-money warrants and decreases with the increase in stock price. Price differences are more pronounced for low stock volatility case than for the high volatility case.

As expected, the method that computes warrant price $w_{W}$ based on the approximation to the 
firm value $V \approx S \cdot N$ does best in the low dilution case. The approximation takes into account only the value of stock and therefore becomes inaccurate when the number of warrants outstanding is relatively large. The choice of approximation to the firm value becomes crucial.

Next, note that even when three different methods provide close results for warrant prices, the volatility of stock returns, $\sigma_{S}$, may be very different from the volatility of the firm value process, $\sigma$. The difference in volatilities is larger for the in-the-money warrants (ITM) and increases with dilution. For the ITM warrant, $S=110$, in the medium dilution case of one warrant for every two outstanding stocks, $\sigma_{S}=25 \%$, but the variance of the firm value is substantially higher at $\sigma=30.35 \%$. When the variance is higher, the difference is even larger: $\sigma_{S}=40 \%$ and $\sigma=46.59 \%$. These differences may be important for studies that measure implied volatility from prices of warrant or rights. Computed volatilities will differ significantly depending on the warrant valuation model employed, especially for a warrant issue with a high degree of dilution. This is also true for short-term deep in-the-money rights issues. If the objective of the study is to measure the volatility of the firm value process, then the warrant valuation algorithm developed in this paper may be used. The algorithm takes into account the dilution caused by the warrant issue and the difference between the stock return volatility and the firm value volatility.

\section{Conclusion}

In this paper I derive a method for pricing warrants and rights using observable variables. The algorithm is based on the variables used in the Black-Scholes option pricing formula, the number of shares outstanding, the number of warrants issued, and the number of shares of stock that each warrant entitles the owner to receive when exercised. The algorithm is based on solving a system 
of equations numerically using readily available software. A proof for the existence of the solution is provided. 


\section{A Appendix}

Proof of Theorem 1. Step 1. In this step we prove that $\forall \sigma \in(0,+\infty) \exists$ unique $V(\sigma) \in(0,+\infty)$

such that

$$
S \cdot N=V-n \cdot \mathcal{W}(V, \tau ; X, \sigma, r, k, N, n)
$$

For convenience define $Z \equiv k V$. Then the first equation of the system becomes

$$
S N=V-n \mathcal{W}(V, \tau ; X, \sigma, r, k, N, n) \Leftrightarrow S N=\frac{Z}{k}-\frac{n}{N+k n} \mathcal{C}(Z, \tau ; N X, \sigma, r) .
$$

Define the distance

$$
d(\sigma, Z) \equiv \frac{Z}{k}-\frac{n}{N+k n} \mathcal{C}(Z, \tau ; N X, \sigma, r)
$$

The distance function has four properties that we need. First, $\forall \sigma>0, \lim _{Z \rightarrow 0} d(\sigma, Z)=0$, because $\lim _{Z \rightarrow 0} \eta_{W}=-\infty$ and $\lim _{Z \rightarrow 0} \mathcal{C}(Z, \bullet)=0$. Next, we use well-known expression for call option delta, $\frac{\partial \mathcal{C}(Z ; \bullet)}{\partial Z}=\Phi\left(\eta_{W}\right) \in(0,1)$, to show that $\forall \sigma>0, d(\sigma, Z)$ is an increasing function of $Z$ :

$$
\frac{\partial}{\partial Z} d(\sigma, Z)=\frac{1}{k}-\frac{n}{N+k n} \Phi\left(\eta_{W}\right)=\frac{1}{k}\left[1-\frac{k n}{N+k n} \Phi\left(\eta_{W}\right)\right]>0, \forall Z
$$

To determine the sign of $d(\sigma, Z)$ note that $\forall \sigma>0$ and $\forall Z>0, \mathcal{C}(Z, \sigma ; \bullet)<Z$ and therefore $\exists \varepsilon>0: \mathcal{C}(Z, \sigma ; \bullet)=Z-\varepsilon$. Then

$$
d(\sigma, Z)=\frac{Z}{k}-\frac{n(Z-\varepsilon)}{N+k n}=\frac{Z N+k n Z-k n Z+k n \varepsilon}{k(N+k n)}=\frac{Z N+k n \varepsilon}{k(N+k n)}>0 .
$$

In sum, the distance function $d(\sigma, Z)$ is continuous in $Z$ (by continuity of $\mathcal{C}(Z, \sigma ; \bullet)$ ), strictly positive, monotonically increasing in $Z$ and $\lim _{Z \rightarrow 0} d(\sigma, Z)=0$. Also, $\forall \sigma>0: d(\sigma, Z) \longrightarrow+\infty$ as $Z \rightarrow+\infty$, because $\mathcal{C}(Z, \sigma ; \bullet) \longrightarrow+\infty$ as $Z \rightarrow+\infty$. The function, therefore, attains any strictly positive value. 
Hence, $\forall \sigma>0, \forall a>0, \exists Z>0: d(\sigma, Z)=a$. Take $a=S N$ and let $V=Z / k$, and conclude that, since $V$ is unique, there is a continuous map $\psi_{1}: \sigma \mapsto V$, which is written as implicit function

$$
0=\Psi_{1}(\sigma, V) \equiv V-n \mathcal{W}(V, \tau ; X, \sigma, r, k, N, n)-S N
$$

The function $\psi_{1}: \sigma \mapsto V$ is increasing: $\forall \sigma>0, \frac{d \psi_{1}}{d \sigma}>0$. The formal argument involves implicit function differentiation,

$$
\frac{d V}{d \sigma}=-\frac{\partial \Psi_{1} / \partial \sigma}{\partial \Psi_{1} / \partial V}=-\frac{-\frac{n}{N+k n} \frac{\partial \mathcal{C}(k V, \sigma ; \bullet)}{\partial \sigma}}{1-\frac{k n}{N+k n} \frac{\partial \mathcal{C}(Z, \sigma ; \bullet)}{\partial Z}}=\frac{n}{N+k n} \frac{\partial \mathcal{C}(k V, \sigma ; \bullet)}{\partial \sigma} /\left[1-\frac{k n}{N+k n} \Phi\left(\eta_{W}\right)\right]>0
$$

The inequality holds true because of the well-known property, $\partial \mathcal{C}(Z, \sigma ; \bullet) / \partial \sigma>0$. However, there is a more intuitive proof that $\psi_{1}$ is increasing in $\sigma$. It is well-known that call price, $\mathcal{C}(Z, \sigma ; \bullet)$, is increasing in $\sigma$. So when $\sigma$ is increased, $\mathcal{W}(\bullet)$ increases and $\Psi_{1}(\sigma, V)$ becomes negative. If $V$ is increased now, then the first term $V$ goes up one-for-one with $V$, but the second term, $n \mathcal{W}(\bullet)$, increases at a slower rate. This is so because $\frac{\partial \mathcal{W}(\bullet)}{\partial V}=\triangle_{W}=\frac{k}{N+k n} \Phi\left(\eta_{W}\right) \in(0,1)$. Hence, $V$ can be increased until the equality $\Psi_{1}(\sigma, V)=0$ is restored. We conclude that $\psi_{1}$ is an increasing function.

Step 2. In this step we prove that $\forall \sigma \in(0,+\infty) \exists V(\sigma) \in(0,+\infty)$ such that $\sigma_{S}=\left(V \triangle_{S} / S\right) \cdot \sigma$. Define function

$$
f(\sigma, V) \equiv \frac{V \triangle_{S}}{S} \sigma=\frac{V}{S} \sigma\left[\frac{N+k n\left[1-\Phi\left(\eta_{W}\right)\right]}{N(N+k n)}\right] .
$$

This function is strictly positive, and continuous in $V$ for all positive $\sigma$. We will show that $f(\sigma, V)$ vanishes as $V \rightarrow 0(\forall \sigma>0)$, and increases without bound as $V \rightarrow+\infty,(\forall \sigma>0)$ :

$$
\begin{aligned}
& V \quad \rightarrow \quad+\infty \Rightarrow \eta_{W} \rightarrow+\infty \Rightarrow \Phi\left(\eta_{W}\right) \rightarrow 1 \Rightarrow f(\sigma, V) \rightarrow+\infty, \forall \sigma>0 \\
& V \quad \rightarrow \quad 0 \Rightarrow \eta_{W} \rightarrow-\infty \Rightarrow \Phi\left(\eta_{W}\right) \rightarrow 0 \Rightarrow f(\sigma, V) \rightarrow 0, \forall \sigma>0
\end{aligned}
$$


The function $f(\sigma, V)$, therefore, attains any positive value. Hence, $\forall \sigma>0, \forall a>0, \exists V>0$ such that $f(\sigma, V)=a$. Take $a=\sigma_{S}$ and conclude that there is a correspondence $\psi_{2}: \sigma \mapsto V$, which is defined by the implicit function

$$
0=\Psi_{2}(\sigma, V) \equiv \frac{V \triangle_{S}}{S} \sigma-\sigma_{S}
$$

Step 3. Now we need to prove that $\psi_{1}: \sigma \mapsto V$ and $\psi_{2}: \sigma \mapsto V$ intersect. It is already established that $\psi_{1}$ is one-to-one and strictly increasing. It may, however, be bounded as $\sigma \rightarrow 0$ or $\sigma \rightarrow+\infty$. Then, to show that intersection exists, we must show that $\psi_{2}$ attains any value in $(0,+\infty)$

As $\sigma$ increases without bound, $\sigma \rightarrow+\infty$, the function $\psi_{2}(\sigma)$ vanishes. It is not bounded from below by some positive constant. The proof is by Reductio ad absurdum. Assume that there exists a strictly positive lower bound $\underline{V_{2}}$ such that $\psi_{2}(\sigma) \in\left(0, \underline{V_{2}}\right)$. Then $\eta_{W}\left(\underline{V_{2}}, \sigma\right)$ can be increased without bound by taking sufficiently large $\sigma$. Then $f(\sigma, V)$ increases without bound and cannot be made equal to fixed $\sigma_{S}$. But we know that for any $\sigma$ we can always find $V$ so that $\Psi_{2}(\sigma, V)=0$ is satisfied. Hence, $V$ is not bounded below by $\underline{V_{2}}$ and $\lim _{\sigma \rightarrow+\infty} V=\lim _{\sigma \rightarrow+\infty} \psi_{2}(\sigma)=0$.

As $\sigma \rightarrow 0$ the function $\psi_{2}(\sigma)$ increases without bound, $\psi_{2}(\sigma) \rightarrow+\infty$. The function is not bounded from above by any positive constant. As $\sigma$ decreases, the term

$$
\left[N+k n\left(1-\Phi\left(\eta_{W}\right)\right)\right] /[N(N+k n)]
$$

remains bounded, hence $f(\sigma, V)$ can be made arbitrarily small and cannot be made equal to fixed $\sigma_{S}$. But we know that for any $\sigma$ we can always find $V$ so that $\Psi_{2}(\sigma, V)=0$ is satisfied. Hence, $V$ is not bounded above and $\lim _{\sigma \rightarrow 0} V=\lim _{\sigma \rightarrow 0} \psi_{2}(\sigma)=+\infty$.

This proves that $\psi_{2}$ attains any value in $(0,+\infty)$. It is also continuous, and $\lim _{\sigma \rightarrow 0} \psi_{2}(\sigma)=$ $+\infty, \lim _{\sigma \rightarrow+\infty} \psi_{2}(\sigma)=0$. Hence, intersection with $\psi_{1}$ exists. 


\section{References}

[1] Black, F. and Scholes, M., 1973, "The pricing of options and corporate liabilities," Journal of Political Economy, 81, 637-659.

[2] Crouhy, Michel, and Dan Galai, 1991, "Common Errors in the Valuation of Warrants and Options on Firms with Warrants," Financial Analysts Journal, vol. 47, no. 5, 89-90.

[3] Galai, Dan, and Mier I. Schneller, 1978, "The Pricing of Warrants and the Value of the Firm," Journal of Finance, 1333-1342.

[4] Hull, John C., 2003, Options, Futures, and Other Derivatives, 5th Edition (Prentice Hall, New York).

[5] Ingersoll, Jonathan E., Jr., 1987, Theory of Financial Decision Making (Rowman \& Littlefield Publishers, Inc.)

[6] Ingersoll, Jonathan E., Jr., 1994, "Financial Instruments and Contracts: Exotic Option Contracts," Mimeo, Yale University, School of Management.

[7] Phelps, Katherine L., William T. Moore, and Rodney L. Roenfeldt, "Equity Valuation Effects of Warrant-Debt Financing," Journal of Financial Research, Summer 1991, 93-103.

[8] Veld, Chris, 2000, "Warrant Pricing: A Review of Empirical Reserch," Working paper, www.ssrn.com

[9] Veld, C. and Verboven, A., 1995, "An empirical analysis of warrant prices versus long term call option prices," Journal of Business Finance and Accounting, pp. 1125-1146. 
TABLE 1. Comparison of Alternative Warrant Valuation Methods.

\begin{tabular}{|c|c|c|c|c|c|c|c|c|}
\hline \multirow[b]{2}{*}{$S$} & \multicolumn{4}{|c|}{ Low Variance, $\sigma_{S}=25 \%$} & \multicolumn{4}{|c|}{ High Variance, $\sigma_{S}=40 \%$} \\
\hline & $w_{\text {Call }}$ & $w_{W}$ & $w_{A}$ & $\sigma$ & $w_{\text {Call }}$ & $w_{W}$ & $w_{A}$ & $\sigma$ \\
\hline \multicolumn{9}{|c|}{ Panel A: Low Dillution, $N=100, n=10$} \\
\hline 75 & 8.86 & 8.05 & 8.81 & $25.82 \%$ & 16.61 & 15.10 & 16.56 & $41.40 \%$ \\
\hline 100 & 23.67 & 21.52 & 23.68 & $26.15 \%$ & 32.60 & 29.64 & 32.57 & $41.48 \%$ \\
\hline 110 & 31.14 & 28.31 & 31.15 & $26.20 \%$ & 39.95 & 36.31 & 39.91 & $41.51 \%$ \\
\hline \multicolumn{9}{|c|}{ Panel B: Medium Dilution, $N=100, n=50$} \\
\hline 75 & 8.86 & 5.90 & 8.65 & $28.72 \%$ & 16.61 & 11.07 & 16.37 & $45.77 \%$ \\
\hline 100 & 23.67 & 15.78 & 23.68 & $30.14 \%$ & 32.60 & 21.73 & 32.40 & $46.51 \%$ \\
\hline 110 & 31.14 & 20.76 & 31.14 & $30.35 \%$ & 39.95 & 26.63 & 39.71 & $46.59 \%$ \\
\hline \multicolumn{9}{|c|}{ Panel C: High Dilution, $N=100, n=100$} \\
\hline 75 & 8.86 & 4.43 & 8.49 & $31.73 \%$ & 16.61 & 8.30 & 16.16 & $50.26 \%$ \\
\hline 100 & 23.67 & 11.84 & 23.61 & $34.17 \%$ & 32.60 & 16.30 & 32.15 & $51.39 \%$ \\
\hline 110 & 31.14 & 15.57 & 31.05 & $34.47 \%$ & 39.95 & 19.97 & 39.42 & $51.46 \%$ \\
\hline
\end{tabular}

Note: This table displays warrant prices computed by three methods: $w_{\text {Call }}$ is warrant price computed according to the Black-Scholes call option valuation formula $(7) ; w_{W}$ corrects for dillution according to (1) and uses $\sigma \approx \sigma_{S}$, and $V \approx S \cdot N ; w_{A}$ is the warrant price and $\sigma$ is the variance of the firm value process computed according to Algorithm 1. Parameters are: $k=1, X=100, \tau=3, r=0.0488$. The warrant prices are computed for two levels of stock volatility, three stock prices, and three different levels of dillution due to warrant exercise. 